\title{
Association of dialysis facility-level hemoglobin measurement and erythropoiesis-stimulating agent dose adjustment frequencies with dialysis facility-level hemoglobin variation: a retrospective analysis
}

Irfan Khan ${ }^{1^{*}}$, Mahesh Krishnan ${ }^{2}$, Anupam Kothawala ${ }^{1}$ and Akhtar Ashfaq ${ }^{1}$

\begin{abstract}
Background: A key goal of anemia management in dialysis patients is to maintain patients' hemoglobin ( $\mathrm{Hb})$ levels consistently within a target range. Our aim in this study was to assess the association of facility-level practice patterns representing $\mathrm{Hb}$ measurement and erythropoiesis-stimulating agent (ESA) dose adjustment frequencies with facility-level $\mathrm{Hb}$ variation.

Methods: This was a retrospective observational database analysis of patients in dialysis facilities affiliated with large dialysis organizations as of July 01, 2006, covering a follow-up period from July 01, 2006 to June 30, 2009. A total of 2,763 facilities representing 436,442 unique patients were included. The predictors evaluated were facility-level $\mathrm{Hb}$ measurement and ESA dose adjustment frequencies, and the outcome measured was facility-level Hb variation.

Results: First to 99th percentile ranges for facility-level $\mathrm{Hb}$ measurement and ESA dose adjustment frequencies were approximately once per month to once per week and approximately once per 3 months to once per 3 weeks, respectively. Facility-level Hb measurement and ESA dose adjustment frequencies were inversely associated with $\mathrm{Hb}$ variation. Modeling results suggested that a more frequent $\mathrm{Hb}$ measurement (once per week rather than once per month) was associated with approximately $7 \%$ to $9 \%$ and $6 \%$ to $8 \%$ gains in the proportion of patients with $\mathrm{Hb}$ levels within $\mathrm{a} \pm 1$ and $\pm 2 \mathrm{~g} / \mathrm{dL}$ range around the mean, respectively. Similarly, more frequent ESA dose adjustment (once per 2 weeks rather than once per 3 months) was associated with approximately $6 \%$ to $9 \%$ and $5 \%$ to $7 \%$ gains in the proportion of patients in these respective $\mathrm{Hb}$ ranges.
\end{abstract}

Conclusions: Frequent $\mathrm{Hb}$ measurements and timely ESA dose adjustments in dialysis patients are associated with lower facility-level $\mathrm{Hb}$ variation and an increase in proportion of patients within \pm 1 and $\pm 2 \mathrm{~g} / \mathrm{dL}$ ranges around the facility-level $\mathrm{Hb}$ mean.

\section{Background}

A central aim of anemia management in dialysis patients is to maintain patients' hemoglobin $(\mathrm{Hb})$ levels consistently within a target range. Anemia management guidelines and protocols thus require appropriate adjustment of erythropoiesis-stimulating agent (ESA) doses in

\footnotetext{
* Correspondence: kirfan@amgen.com

'Amgen Inc., One Amgen Center Drive, Thousand Oaks, CA, 91320-1799, USA

Full list of author information is available at the end of the article
}

response to $\mathrm{Hb}$ deviations relative to the target range. There are varied opinions among clinicians, however, regarding practice patterns that help achieve this objective [1-7].

The current US Food and Drug Administrationapproved ESA label recommends an $\mathrm{Hb}$ target range of 10 to $12 \mathrm{~g} / \mathrm{dL}$ when treating dialysis patients $[8,9]$, which represents a slightly broader range than the 11 to $12 \mathrm{~g} / \mathrm{dL}$ $\mathrm{Hb}$ target currently recommended by the National Kidney Foundation [10]. Recent data from randomized clinical

\section{() Biomed Central}


trials (RCTs) have suggested no benefit and raised concerns regarding deleterious effects observed with $\mathrm{Hb}$ targets of 13-15 g/dL in patients with chronic kidney disease who are treated with ESAs [11-14]. At the same time, however, there are adverse consequences associated with low Hb levels [15-21]. Thus, at the level of individual dialysis provider, $\mathrm{Hb}$ target ranges have been influenced by the need to balance these opposing risks with the benefits of achieving $\mathrm{Hb}$ levels within the 10 to $12 \mathrm{~g} / \mathrm{dL}$ range.

Recently, the Centers for Medicare and Medicaid Services proposed a rule for the End-Stage Renal Disease Prospective Payment System that includes a payment penalty for dialysis facilities that maintain more than a specified proportion of patients in $\mathrm{Hb}<10$ or $>12 \mathrm{~g} / \mathrm{dL}$ ranges [22]. It is therefore helpful for clinicians to understand the nature of anemia management protocols that could maximize the number of patients within the desired $\mathrm{Hb}$ target range. Our aim in this study was to use the historical cross-sectional and longitudinal variation in the facility-level practice patterns of $\mathrm{Hb}$ measurement and ESA dose adjustment frequencies to study their association with the facility-level $\mathrm{Hb}$ variation, which is a key summary measure of a facility's ability to maintain patients within a given $\mathrm{Hb}$ target range.

\section{Methods}

A retrospective observational database was constructed from electronic patient-level data contributed by large dialysis organizations (LDOs) in the United States. An LDO was defined as a chain of $>500$ affiliated dialysis centers. All data were de-identified in accordance with the Health Insurance Portability and Accountability Act Privacy Rule for limited use datasets; thus no additional institutional review board approval was sought for this study. This database has been described in detail in a previous publication [23]. Data from July 2006 to June 2009 (36 months) were extracted from dialysis centers affiliated with an LDO as of July 01, 2006. A study time period of July 2006 to June 2009 was chosen because it coincided with a significant longitudinal variation in the facility-level $\mathrm{Hb}$ variation, $\mathrm{Hb}$ measurement frequency, and ESA dose adjustment frequency. This longitudinal variation was influenced in part by regulatory and reimbursement changes for ESAs during this time. Only hemodialysis (HD) patients with $>30$ days of data during this 36-month interval were selected. Included HD patients represented only the thrice-weekly in-center HD modality, while other variants of HD treatment (daily, home, nocturnal, etc.) were not included in the cohort. Although ESA administration was not considered an inclusion/exclusion criterion, the ESA type variable in the analysis dataset and available LDO protocol information indicated that all ESA administrations in the final study cohort were in the form of intravenous epoetin alfa.

The dialysis facility was regarded as the unit of analysis. For each dialysis facility, the following sets of variables were calculated for each month during the study. They were (1) $\mathrm{Hb}$ variation, defined as the SD of all $\mathrm{Hb}$ measurements across all patients; (2) the number of patient-days, defined as the summation across all patients of the following measure: date of last patientlevel dialysis session - date of first patient-level dialysis session $+(7 / 3)$, with $7 / 3$ applied to adjust for the follow-up truncation at the last observation in a month, assuming the average sequence of dialysis sessions was 3 times per week (TIW) for HD patients; (3) $\mathrm{Hb}$ measurement frequency, defined as the number of $\mathrm{Hb}$ measurements across all patients divided by the number of patient-days/30; and (4) ESA dose adjustment frequency, defined as the number of ESA dose adjustments across all patients divided by the number of patient-days $/ 30$.

Only persistent dose changes were regarded as true clinical dose adjustments (i.e., those that appeared as $a-a-a-b-b-b$, as opposed to $a-a-a-b-a-a$, over a TIW sequence). Dose changes were considered to be persistent if they lasted for at least 3 TIW sessions for nonzero doses and at least 9 TIW sessions for zero doses. The longer time requirement for zero-dose adjustments helped reduce potential misclassification of intentional clinical adjustments to zero doses with hospitalization events. We validated the methodology with data from patients for whom hospitalization information was available (approximately $50 \%$ of the study cohort). The validation step indicated that this methodology would result in a very low proportion (approximately $0.3 \%$ ) of dose adjustments that could be potentially misclassified. We believe that this level of error is unlikely to cause a meaningful impact on the results. The algorithm adopted for identifying dose changes considered the entire patient-level longitudinal data before further categorization by calendar months. The calendar month boundaries thus did not interfere with the definition of a dose change.

We summarized the aggregate variation in facility-level $\mathrm{Hb}$ variation, $\mathrm{Hb}$ measurement frequency, and ESA dose adjustment frequency by pooling these measurements across all facility-month combinations and calculating basic descriptive statistics. A continuous distribution curve for these measures was created by calculating the percentage of facility-months within specific intervals. By adopting a method similar to one in a previous publication [23], we produced a smooth approximation of the distribution curve to avoid unnecessary attention to density fluctuations in low-prevalence buckets. The longitudinal variation in these measures was summarized by calculating the mean $(95 \% \mathrm{CI})$ values by each month 
across all facilities. The number of patient-days was introduced as a weight in these calculations.

We studied associations between facility-level $\mathrm{Hb}$ variation and $\mathrm{Hb}$ measurement frequency and between facility-level $\mathrm{Hb}$ variation and ESA dose adjustment frequency by using a mixed-modeling regression approach that partitioned cross-sectional and longitudinal information in the data [24-26]. This modeling framework was adopted to address the unavailability of facility-level characteristics, which was one of the main limitations of the analysis dataset. When conclusions regarding associations in this modeling framework are drawn from the longitudinal part of the model, the same facility will effectively act as its own control over time, and the primary source of bias will be limited to systematic longitudinal changes in aggregate facility-level characteristics. The technical details and underlying rationale behind this are discussed more fully in previous work using these models [24-26].

We considered 2 separate models for studying the associations between facility-level $\mathrm{Hb}$ variation and $\mathrm{Hb}$ measurement frequency and between facility-level $\mathrm{Hb}$ variation and ESA dose adjustment frequency due to the relatively high collinearity between $\mathrm{Hb}$ measurement and ESA dose adjustment frequencies. An additional reason for considering 2 separate models was that a model which simultaneously considered both these factors could introduce a conceptual possibility that the benefit from these factors in terms of their implications on $\mathrm{Hb}$ variation could be regarded as additive and can accrue independently. Though the benefit from $\mathrm{Hb}$ measurement frequency can be considered to accrue independently, the same cannot be said about the ESA dose adjustment frequency since any practical anemia management protocol would consider $\mathrm{Hb}$ measurement frequency to exceed the ESA dose adjustment frequency. The fixed- and random-effects part of these mixed models included an identical set of parameters representing an intercept term, a linear cross-sectional slope parameter, and a linear longitudinal slope parameter. The number of patient-days was introduced as a weight in the preceding models. The mixed models were implemented using Proc MIXED in SAS (SAS Institute Inc., Cary, NC, USA). All analyses were performed in SAS, version 9.1.3.

We consider the model relating facility-level $\mathrm{Hb}$ variation and $\mathrm{Hb}$ measurement frequency as not being influenced by an endogeneity problem due to omission of the number of $\mathrm{Hb}$ measurements from the model. This is because we defined $\mathrm{Hb}$ variation in terms of $\mathrm{SD}$. The expectation of $\mathrm{Hb}$ variation is therefore independent of sampling more or less $\mathrm{Hb}$ observations, which would influence the precision of estimating the SD. We would highlight that we have not defined $\mathrm{Hb}$ variation as the precision of SD, but SD itself, which is a summary measure of the width of the facility-level $\mathrm{Hb}$ distribution curve.

Please see the Technical Appendix (Additional file 1) for further information regarding model formulation.

\section{Results}

A total of 2,763 facilities were included in the analysis. They represented all LDO-affiliated facilities as of July 01,2006 , and accounted for approximately $60 \%$ of all dialysis facilities in the United States at that time. Data from 2,734 facilities ( $99 \%$ of the original cohort) were available for all 36 months of follow-up, with the remaining facilities (1\%) lost to follow-up owing to closure. The total number of unique patients and facilitymonth combinations represented in the analysis dataset during the 36-month study period were 436,442 and 98,717 , respectively.

Figure 1A summarizes the distribution of facility-level $\mathrm{Hb}$ variation across facility-months. The mean facilitylevel $\mathrm{Hb}$ variation was $1.34 \mathrm{~g} / \mathrm{dL}$, with 1st and 99th percentiles ranging from 0.92 to $1.88 \mathrm{~g} / \mathrm{dL}$. Under the assumption of normality for $\mathrm{Hb}$ distribution curve at any point in time (corroborated in previous analyses considering a large cohort of patients $[23,27]$ ), the proportion of patients with $\mathrm{Hb}$ within \pm 1 and $\pm 2 \mathrm{~g} / \mathrm{dL}$ around the mean was higher for the 1st percentile behavior as compared with the 99th by approximately $32 \%$ and $26 \%$, respectively (the percentage point difference represents $\mathrm{p} 99$ - p1, where $\mathrm{p} 99$ and $\mathrm{p} 1$ denote the proportion of patients in specific ranges for facility-months representing the 99th and 1st percentile behavior, respectively). The distribution of facility-level $\mathrm{Hb}$ variation was largely symmetrical about the mean with a slight positive skew (skewness factor, 0.49).

Figures $1 \mathrm{~B}$ and $1 \mathrm{C}$ summarize the distributions of facility-level $\mathrm{Hb}$ measurement and ESA dose adjustment frequencies across facility-months. The mean facility-level $\mathrm{Hb}$ measurement frequency was 2.65 per month (approximately once per 1.5 weeks), with 1st and 99th percentiles ranging from 1.10 to 4.65 per month (approximately once per month to once per week). The distribution of facilitylevel $\mathrm{Hb}$ measurement frequency was bimodal with peaks at approximately 1.97 and 3.74 per month (approximately once per 2 weeks and once per 1 week). The distribution of facility-level $\mathrm{Hb}$ measurement frequency had a positive skew (skewness factor, 0.70 ), indicating a small proportion of facility-months with $\mathrm{Hb}$ measurement frequency significantly exceeding the mean. The mean of facility-level ESA dose adjustment frequency was 0.85 per month (approximately once per 5 weeks) with 1st and 99th percentiles ranging from 0.31 to 1.50 per month (approximately once per 3 months to once per 3 weeks). The distribution of facility-level ESA dose adjustment frequency was largely 

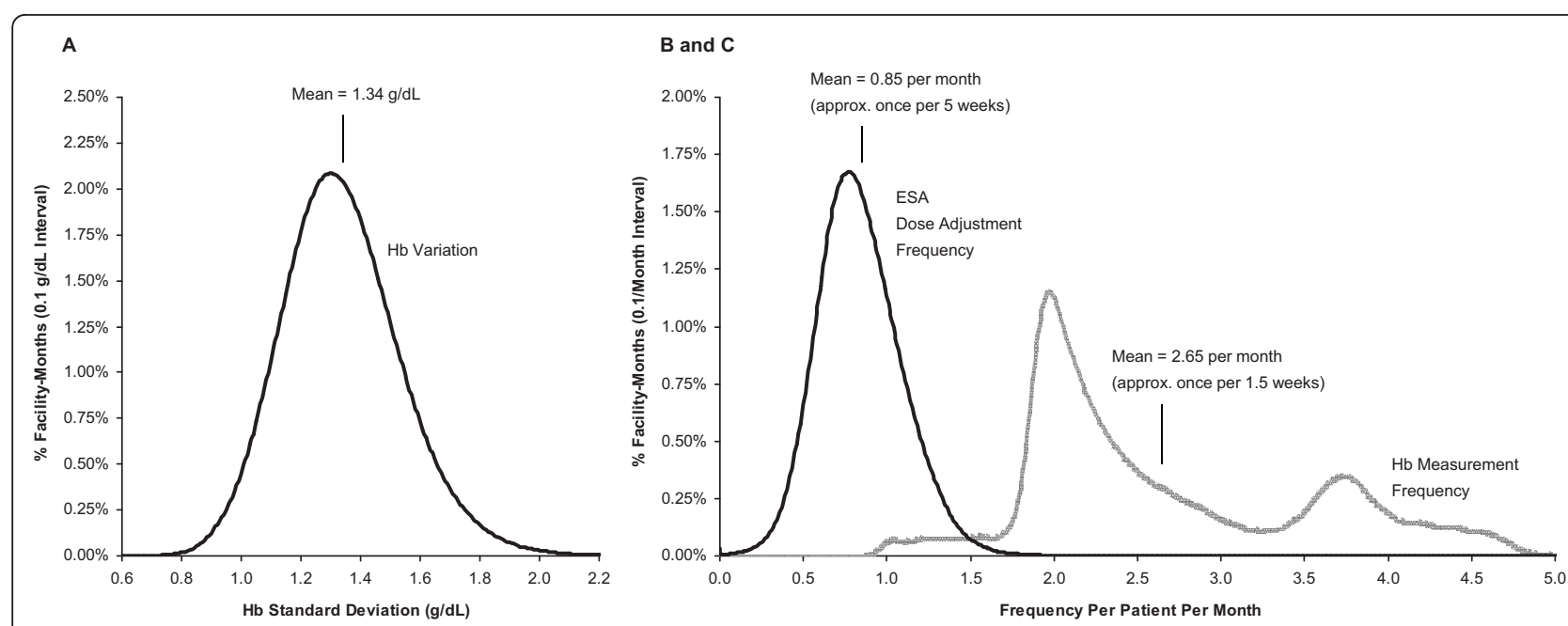

Figure 1 Distribution of study variables across facility-months. (A) Hb variation, (B) Hb measurement frequency, and (C) ESA dose adjustment frequency. ESA = erythropoiesis-stimulating agent; $\mathrm{Hb}=$ hemoglobin.

symmetrical about the mean with a slight positive skew (skewness factor, 0.39). There was a significant positive correlation between the distributions of facility-level $\mathrm{Hb}$ measurement and ESA dose adjustment frequencies with an estimated Pearson Correlation Coefficient of 30.7\% $(P<0.001)$.

Figure 2 summarizes the longitudinal variation in the mean values of facility-level $\mathrm{Hb}$ variation, $\mathrm{Hb}$ measurement frequency, and ESA dose adjustment frequency. Mean facility-level $\mathrm{Hb}$ variation (Figure 2A) decreased from $1.41 \mathrm{~g} / \mathrm{dL}$ ( $95 \% \mathrm{CI}, 1.40-1.42 \mathrm{~g} / \mathrm{dL}$ ) in July 2006 to $1.24 \mathrm{~g} / \mathrm{dL}$ (95\% CI, 1.23-1.24 g/dL) in June 2009. Under the assumption of normality for $\mathrm{Hb}$ distribution curve at any time point, this indicated that on average a facility's ability to maintain patients within \pm 1 and $\pm 2 \mathrm{~g} / \mathrm{dL}$ around the mean during the study period increased by approximately $6 \%$ and $5 \%$, respectively. Mean facilitylevel $\mathrm{Hb}$ measurement frequency (Figure 2B) increased from 2.50 per month (95\% CI, 2.47-2.54 per month) in July 2006 to 3.12 per month (95\% CI, 3.08-3.16 per month) in June 2009, while mean facility-level ESA dose adjustment frequency (Figure 2C) increased from 0.76 per month (95\% CI, 0.75-0.77 per month) in July 2006 to 1.01 per month (95\% CI, $1.00-1.02$ per month) in June 2009.

Table 1 provides a summary of the estimates for fixedeffect parameters for the previously described models that evaluated the associations between facility-level $\mathrm{Hb}$ variation and $\mathrm{Hb}$ measurement frequency and between facility-level $\mathrm{Hb}$ variation and ESA dose adjustment frequency. For both models, the estimates for intercept, cross-sectional parameter, and longitudinal parameter

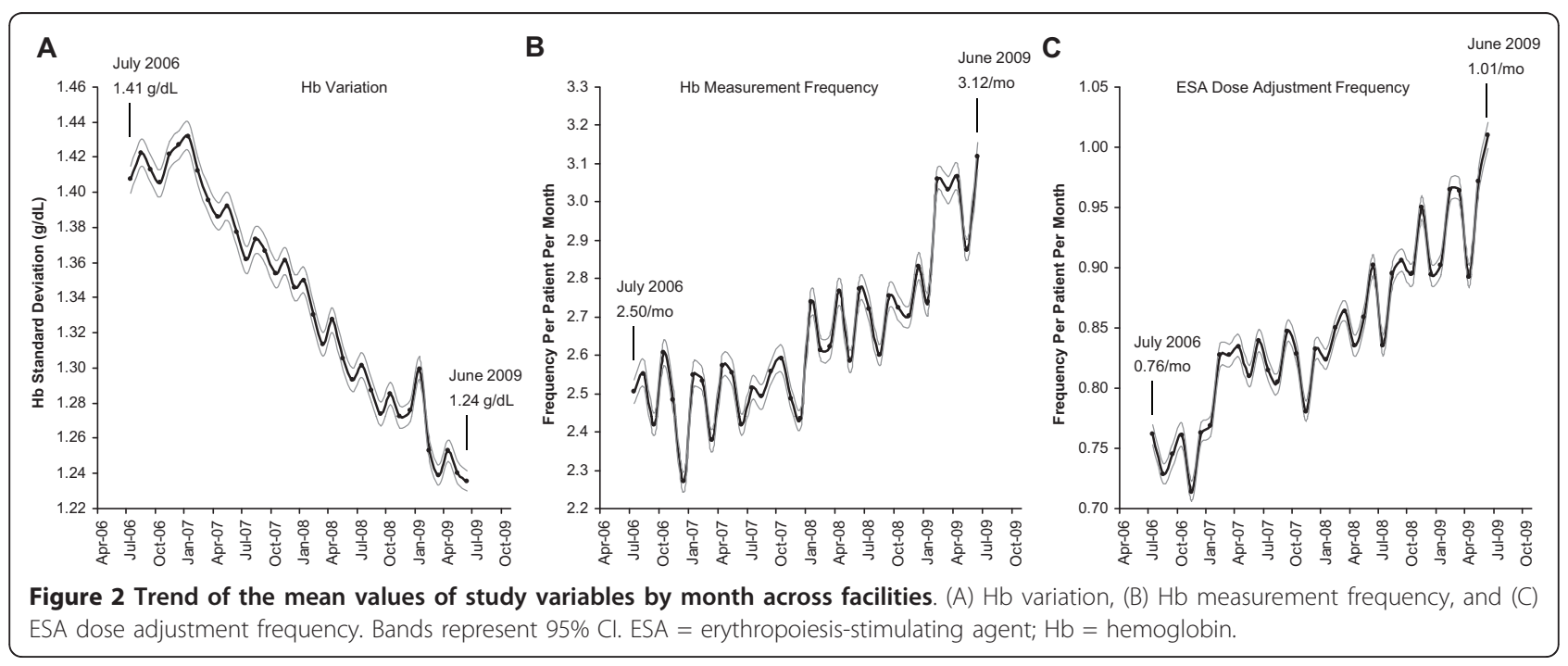


Table 1 Fixed-effect parameter estimates for mixed models

\begin{tabular}{lccc}
\hline & \multicolumn{3}{c}{ Fixed-Effect Parameter Estimates* $(95 \% \mathrm{Cl})$} \\
\cline { 2 - 4 } Model & Intercept & Cross-Sectional Parameter & Longitudinal Parameter \\
Facility-level $\mathrm{Hb}$ variation and $\mathrm{Hb}$ measurement frequency & 1.558 & -0.083 & -0.066 \\
& $(1.543$ to 1.573$)$ & $(-0.089$ to -0.078$)$ & $(-0.070$ to -0.062$)$ \\
Facility-level $\mathrm{Hb}$ variation and ESA dose adjustment frequency & 1.443 & -0.133 & -0.096 \\
& $(1.427$ to 1.460$)$ & $(-0.154$ to -0.112$)$ & $(-0.104$ to -0.088$)$ \\
\hline
\end{tabular}

These estimates assess the associations between facility-level $\mathrm{Hb}$ variation and $\mathrm{Hb}$ measurement frequency and between facility-level $\mathrm{Hb}$ variation and ESA dose adjustment frequency.

ESA = erythropoiesis-stimulating agent; $\mathrm{Hb}=$ hemoglobin.

${ }^{*}$ All $P<0.001$.

were significant $(P<0.001)$. The estimates for cross-sectional and longitudinal parameters for both models were negative, which indicated a consistent negative association between facility-level $\mathrm{Hb}$ variation and $\mathrm{Hb}$ measurement frequency and between facility-level $\mathrm{Hb}$ variation and ESA dose adjustment frequency. The magnitude of associations as informed by the cross-sectional and longitudinal parameters was not markedly different for both of these models. Based on the mean values of $\mathrm{Hb}$ measurement and ESA dose adjustment frequencies, the difference between cross-sectional and longitudinal parameter estimates translated to a relatively low facility-level $\mathrm{Hb}$ variation difference of approximately $0.03 \mathrm{~g} /$ $\mathrm{dL}$ to $0.05 \mathrm{~g} / \mathrm{dL}$ as measured by the standard deviation. Formal testing, however, indicated that the cross-sectional and longitudinal parameters were statistically different from one another with a $P$-value for the difference $<0.001$ for both models.

Figures $3 \mathrm{~A}$ and $3 \mathrm{~B}$ provide a graphical summary of the cross-sectional and longitudinal associations between facility-level $\mathrm{Hb}$ variation and $\mathrm{Hb}$ measurement frequency and between facility-level $\mathrm{Hb}$ variation and ESA dose adjustment frequency. These results indicate that under the assumption of normality for $\mathrm{Hb}$ distribution curve at any time point, an increase in $\mathrm{Hb}$ measurement frequency from once per month to once per week (from approximately 1st to 99th percentile behavior) was associated with an increase in the proportion of patients with $\mathrm{Hb}$ within the \pm 1 and $\pm 2 \mathrm{~g} / \mathrm{dL}$ around the mean by approximately 9 and 8 percentage points, respectively, according to the cross-sectional model, and by approximately 7 and 6 percentage points, respectively, according to the longitudinal model. Similarly, an increase in ESA dose adjustment frequency from once per 3 months to once per 3 weeks (from approximately 1 st to 99th percentile behavior) was associated with an increase in the proportion of patients in these ranges by approximately 5 and 4 percentage points, respectively, according to the cross-sectional model, and by approximately 4 and 3 percentage points, respectively, according to the longitudinal model. When we modeled an increase in ESA dose adjustment frequency from once

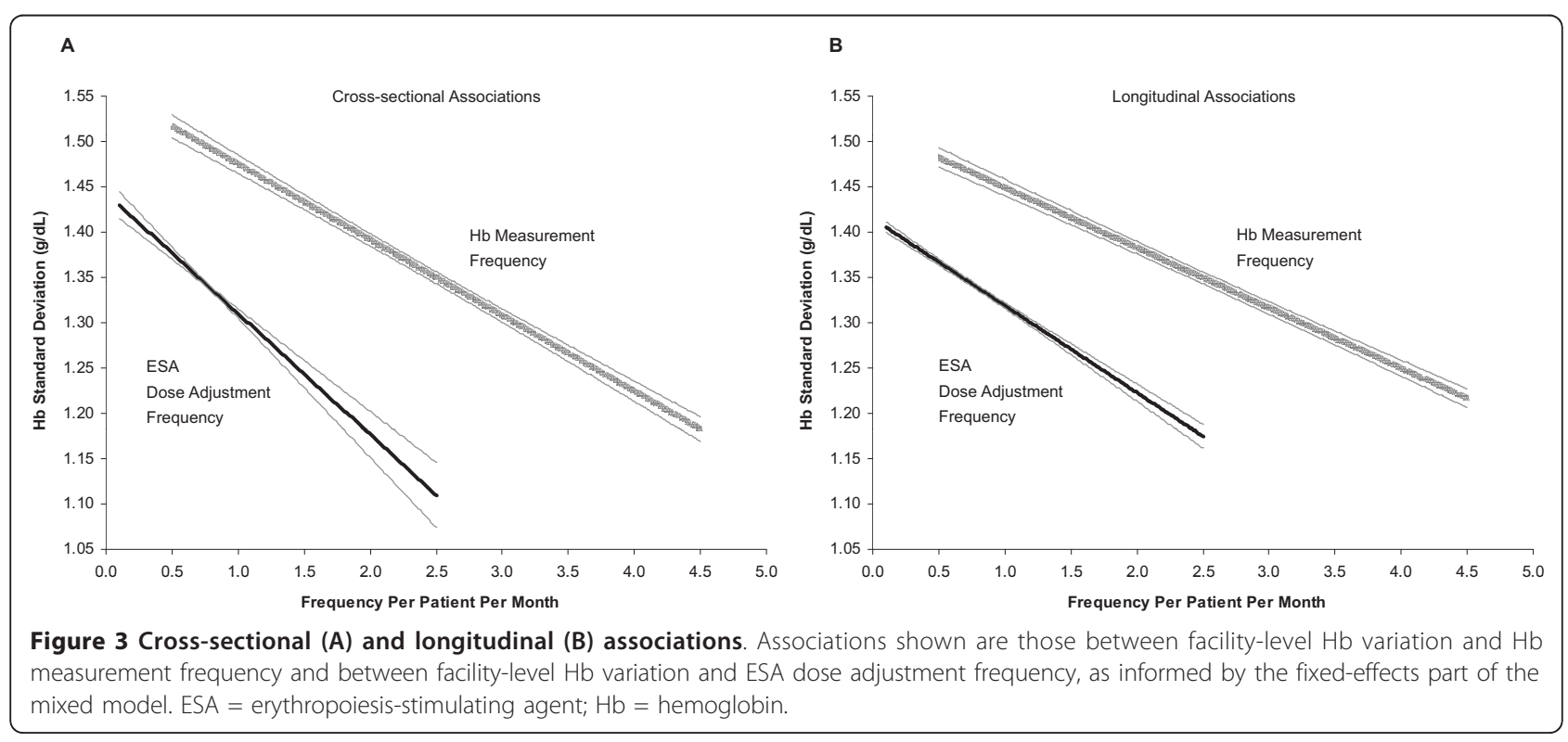


per 3 months to once per 2 weeks, the results were more consistent with the modeled increase in $\mathrm{Hb}$ measurement frequency, and the associated increases in the proportion of patients in these ranges were 9 and 7 percentage points, respectively, according to the cross-sectional model, and 6 and 5 percentage points, respectively, according to the longitudinal model.

\section{Discussion}

We can suggest plausible mechanisms that may be responsible for driving the observed variation in facilitylevel anemia management practice patterns and their association with the facility-level $\mathrm{Hb}$ variation reported in this study. The relatively wide variations observed in facility-level $\mathrm{Hb}$ measurement and ESA dose adjustment frequencies suggest that physicians exercise a significant level of control over these practice patterns in their individual units. Thus, facilities with a higher rate of $\mathrm{Hb}$ monitoring may represent physicians who value an assessment of both point-in-time $\mathrm{Hb}$ measurement as well as the trend. Assessment of an $\mathrm{Hb}$ trend can be helpful; if the trajectory of Hb levels is suggestive of a systematic decline despite stable ESA dosing levels (potentially due to unanticipated changes in a patient's underlying clinical situation driven by concurrent clinical factors and a high level of comorbidity burden), protocols that encourage timely upward adjustment of ESA doses will be associated with better $\mathrm{Hb}$ control. Similarly, if the reverse situation occurs, ESA dosing protocols that encourage a timely reduction or temporary discontinuation of ESAs will result in significantly better control in managing unintended excursions towards the higher $\mathrm{Hb}$ ranges. If ESA doses are temporarily withheld to manage high $\mathrm{Hb}$ level excursions, the practice of frequent $\mathrm{Hb}$ monitoring also allows for a timely re-initiation of treatment before $\mathrm{Hb}$ levels decrease too much.

When the range of facility-level $\mathrm{Hb}$ variation from modeled scenarios was compared with the range represented in the 99th to 1st percentile behavior, it indicated that practice patterns considered in the current study represented only $20 \%$ to $30 \%$ of the potential for reducing the facility-level $\mathrm{Hb}$ variation. This is to be expected because there are a number of other unrelated factors and practice patterns that could influence the facility-level $\mathrm{Hb}$ variation. For example, one such practice pattern is the detailed nature of ESA dose adjustments as a function of specific Hb levels. Similarly, iron administration and iron indices can also influence response to erythropoietin: intravenous iron supplementation is independently associated with the risk of exceeding targeted $\mathrm{Hb}$ levels [28] and changes in intravenous iron administration have been associated with hemoglobin cycling [3]. These factors, though critically important in terms of how they could influence the facility-level $\mathrm{Hb}$ variation, are outside the scope of the current study.

The bimodal distribution of facility-level $\mathrm{Hb}$ measurement frequency reflects the fact that the most popular protocols concerning $\mathrm{Hb}$ measurement frequencies were either once per week or once per 2 weeks, with once per 2 weeks being the more prevalent protocol. The increase in mean facility-level $\mathrm{Hb}$ measurement frequency seen in Figure 2B reflects a systematic practice pattern shift to monitoring $\mathrm{Hb}$ levels more frequently, a behavior that was likely partially driven by changes in the regulatory and reimbursement environment concerning ESA treatment [23]. The alternating nature of mean facility-level $\mathrm{Hb}$ measurement frequency by month seen in Figure 2B could be the result of $\mathrm{Hb}$ measurement frequency being defined in this analysis at a monthly level, whereas the $\mathrm{Hb}$ measurement protocols that were actually being adopted by the dialysis facilities were defined in terms of time intervals counted by weeks. The increase in mean facility-level ESA dose adjustment frequency seen in Figure $2 \mathrm{C}$ reflected a systematic practice pattern shift towards adjusting ESA doses more frequently during the study period, from a frequency of approximately once per 6 weeks to once per 4 weeks, a behavior that was likely driven by the need for maintaining patients' Hb levels within a narrower target range. The same alternating pattern of mean facility-level ESA dose adjustment frequency by month was observed in Figure $2 \mathrm{C}$ with an underlying explanation similar to the trend for mean facility-level $\mathrm{Hb}$ measurement frequency in Figure 2B.

Our method of simultaneously modeling cross-sectional and longitudinal associations in the data is similar to that described elsewhere [24-26]. Details concerning the underlying methodology suggest that the same facility will effectively act as its own control over time in the part of the model informing longitudinal associations. Thus, the longitudinal part of the model helps offset the lack of facility-level covariates, which is one of the main limitations of our analysis data set. The primary source of bias in this case will be the systematic changes in aggregate facility-level characteristics, such as mean demographics and comorbidities, over time. However, these aggregate characteristics change over a much longer time horizon compared with the changes in the practice patterns considered in this study [29]. The cross-sectional and longitudinal parameter estimates were not markedly different from one another, with the difference translating to a facility-level $\mathrm{Hb}$ variation difference of approximately 0.03 to $0.05 \mathrm{~g} / \mathrm{dL}$ as measured by the standard deviation, which could be considered as a value of low practical significance. This suggests that even a purely cross-sectional analysis for assessing these associations may be considered as being somewhat 
moderated from confounding influences. This type of moderation in confounding when an individual facility (as opposed to an individual patient) is regarded as the unit of analysis has been argued in previous studies that have used this approach in the context of dialysis patients $[30,31]$ as well as other settings [32]. However, this perspective should be considered in light of results from formal statistical testing which indicated that the cross-sectional and longitudinal parameters were statistically different from one another, thus technically supporting the design of the current model simultaneously considering cross-sectional and longitudinal effects. We regard the facility-level framework adopted in this analysis, together with the modeling framework that partitioned the cross-sectional and longitudinal variation in the data, as methodologic advantages that could moderate the burden of confounding factors.

There are a number of important limitations of our study that warrant careful consideration. It is possible that the set of studied facility-level practice patterns did not exert a direct causal impact on $\mathrm{Hb}$ variation, but were surrogates for diligent anemia management that could have resulted in a simultaneously elevated likelihood of the implementation of the described practice patterns as well as a reduction in $\mathrm{Hb}$ variation owing to other unrelated factors. These unrelated factors, for example, could represent independent facility-level practice patterns implemented to address the need for managing $\mathrm{Hb}$ levels within a narrower target range over time. An important limitation of the analysis dataset was the unavailability of facility-level demographic characteristics or comorbidity information. However, we have provided a summary description of how the adopted modeling framework could have moderated this limitation. The modeling framework was able to effectively utilize the main strengths of the database, which included the availability of consistent longitudinal data at a patient-level and the ability to map patients to individual dialysis facilities. Other important limitations of the study include the fact that only HD patients within the LDO segment receiving intravenous ESA were represented, which limits the extrapolation of results to other patient and dialysis provider segments. Additionally, patients included in the analysis received only intravenous epoetin alfa and, consequently, these results should be considered valid only for this drug and route of administration. In addition, impact on facility-level $\mathrm{Hb}$ variation due to potential changes in patient-level iron markers and intravenous iron administration were not captured in the analysis due to unavailability of this data. We have implicitly assumed that the longitudinal changes in the $\mathrm{Hb}$ distribution curve at the facility-level during the study time period were primarily influenced by changes in practice patterns concerning $\mathrm{Hb}$ measurement and ESA dose adjustment administration frequencies.

\section{Conclusions}

This study offers useful hypotheses regarding the nature of anemia management practice patterns in dialysis patients that could result in lower facility-level $\mathrm{Hb}$ variation and an increase in the proportion of patients within \pm 1 and $\pm 2 \mathrm{~g} / \mathrm{dL}$ ranges around the facility-level $\mathrm{Hb}$ mean. In particular, this study suggests that the practice patterns of frequent $\mathrm{Hb}$ measurements and timely adjustments of ESA doses could be helpful in attaining this objective.

\section{Additional material}

Additional file 1: Technical Appendix. This appendix contains additional description regarding the model formulation, covariance structure, parameter estimation, and confidence intervals. The appendix also contains residual diagnostics for assessing model adequacy and results from an unweighted analysis.

\section{Acknowledgements and funding}

The authors would like to thank Dikran Toroser and Yeshi Mikyas of Amgen Inc. and Margit Rezabek (services retained by Amgen Inc.) for editorial support. This study was funded by Amgen Inc.

\section{Author details}

'Amgen Inc., One Amgen Center Drive, Thousand Oaks, CA, 91320-1799, USA. ${ }^{2}$ DaVita Inc., 1551 Wewatta St., Denver, CO, 80202, USA.

\section{Authors' contributions}

IK and AK analyzed the data in this study, and all authors interpreted the data. All authors contributed substantially to the writing of this manuscript and read and approved the final manuscript.

\section{Competing interests}

Drs. Khan and Ashfaq and Mr. Kothawala are employees of Amgen Inc. Dr. Krishnan is a former employee of Amgen Inc. and has also served on advisory boards and speaker bureaus for Amgen Inc.

Received: 12 December 2010 Accepted: 20 May 2011

Published: 20 May 2011

\section{References}

1. Chan K, Moran J, Hlatky M, Lafayette R: Protocol adherence and the ability to achieve target haemoglobin levels in haemodialysis patients. Nephrol Dial Transplant 2009, 24(6):1956-1962.

2. Chan KE, Lafayette RA, Whittemore AS, Hlatky MA, Moran J: Facility factors dominate the ability to achieve target haemoglobin levels in haemodialysis patients. Nephrol Dial Transplant 2008, 23(9):2948-2956.

3. Fishbane S, Berns JS: Hemoglobin cycling in hemodialysis patients treated with recombinant human erythropoietin. Kidney Int 2005, 68(3):1337-1343.

4. Fishbane S, Berns JS: Evidence and implications of haemoglobin cycling in anaemia management. Nephrol Dial Transplant 2007, 22(8):2129-2132.

5. Kalantar-Zadeh K, Aronoff GR: Hemoglobin variability in anemia of chronic kidney disease. J Am Soc Nephrol 2009, 20(3):479-487.

6. Miskulin DC, Weiner DE, Tighiouart $H$, Ladik V, Servilla K, Zager PG, Martin A, Johnson HK, Meyer KB: Computerized decision support for EPO dosing in hemodialysis patients. Am J Kidney Dis 2009, 54(6):1081-1088. 
7. Singh AK, Milford E, Fishbane S, Keithi-Reddy SR: Managing anemia in dialysis patients: hemoglobin cycling and overshoot. Kidney Int 2008, 74(5):679-683

8. $\quad$ Aranesp $^{\circledR}$ (darbepoetin alfa): Full Prescribing Information, Amgen, Inc., Thousand Oaks, CA: 2010.

9. Epogen ${ }^{\circledR}$ (epoetin alfa): Full Prescribing Information, Amgen, Inc., Thousand Oaks, CA; 2010.

10. KDOQI clinical practice guideline and clinical practice recommendations for anemia in chronic kidney disease: 2007 update of hemoglobin target. Am J Kidney Dis 2007, 50(3):471-530.

11. Besarab A, Bolton WK, Browne JK, Egrie JC, Nissenson AR, Okamoto DM, Schwab SJ, Goodkin DA: The effects of normal as compared with low hematocrit values in patients with cardiac disease who are receiving hemodialysis and epoetin. N Engl J Med 1998, 339(9):584-590.

12. Drueke TB, Locatelli F, Clyne N, Eckardt KU, Macdougall IC, Tsakiris D, Burger $\mathrm{HU}$, Scherhag A: Normalization of hemoglobin level in patients with chronic kidney disease and anemia. N Engl J Med 2006, 355(20):2071-2084.

13. Pfeffer MA, Burdmann EA, Chen CY, Cooper ME, de Zeeuw D, Eckardt KU, Feyzi JM, Ivanovich P, Kewalramani R, Levey AS, Lewis EF, McGill JB, McMurray JJ, Parfrey P, Parving HH, Remuzzi G, Singh AK, Solomon SD, Toto R: A trial of darbepoetin alfa in type 2 diabetes and chronic kidney disease. N Engl J Med 2009, 361(21):2019-2032.

14. Singh AK, Szczech $L$, Tang KL, Barnhart H, Sapp S, Wolfson M, Reddan D: Correction of anemia with epoetin alfa in chronic kidney disease. $N$ Engl J Med 2006, 355(20):2085-2098.

15. Collins AJ, Li S, St Peter W, Ebben J, Roberts T, Ma JZ, Manning W: Death, hospitalization, and economic associations among incident hemodialysis patients with hematocrit values of 36 to 39\%. J Am Soc Nephrol 2001, 12(11):2465-2473.

16. Foley RN, Parfrey PS, Harnett JD, Kent GM, Murray DC, Barre PE: The impact of anemia on cardiomyopathy, morbidity, and mortality in end-stage renal disease. Am J Kidney Dis 1996, 28(1):53-61.

17. Lowrie EG, Huang $W H$, Lew NL, Liu Y: The relative contribution of measured variables to death risk among hemodialysis patients. In Death on Hemodialysis: Preventable or Inevitable Edited by: Hingman FE. Boston MA: Kluwer Academic Publishers 1994, 121-141.

18. Ma JZ, Ebben J, Xia H, Collins AJ: Hematocrit level and associated mortality in hemodialysis patients. J Am Soc Nephrol 1999, 10(3):610-619.

19. Ofsthun N, Labrecque J, Lacson E, Keen M, Lazarus JM: The effects of higher hemoglobin levels on mortality and hospitalization in hemodialysis patients. Kidney Int 2003, 63(5):1908-1914.

20. Regidor DL, Kopple JD, Kovesdy CP, Kilpatrick RD, McAllister CJ, Aronovitz J, Greenland S, Kalantar-Zadeh K: Associations between changes in hemoglobin and administered erythropoiesis-stimulating agent and survival in hemodialysis patients. J Am Soc Nephrol 2006, 17(4):1181-1191.

21. Roberts TL, Foley RN, Weinhandl ED, Gilbertson DT, Collins AJ: Anaemia and mortality in haemodialysis patients: interaction of propensity score for predicted anaemia and actual haemoglobin levels. Nephrol Dial Transplant 2006, 21(6):1652-1662.

22. Centers for Medicare \& Medicaid Services: Medicare program; end-stage renal disease quality incentive program. In Final rule Edited by: Services DoHaH. Federal Register 2011, 76:628-646.

23. Spiegel DM, Khan I, Krishnan M, Mayne TJ: Changes in hemoglobin leve distribution in US dialysis patients from June 2006 to November 2008. Am J Kidney Dis 2010, 55(1):113-120.

24. Caselli RJ, Dueck AC, Osborne D, Sabbagh MN, Connor DJ, Ahern GL, Baxter LC, Rapcsak SZ, Shi J, Woodruff BK, Locke DE, Snyder CH, Alexander GE, Rademakers R, Reiman EM: Longitudinal modeling of agerelated memory decline and the APOE epsilon4 effect. $N$ Engl J Med 2009, 361(3):255-263.

25. Fitzmaurice GM, Laird NM, Ware JH: Applied Longitudinal Analysis New York, NY: John Wiley and Sons; 2004

26. Ware JH, Dockery DW, Louis TA, Xu XP, Ferris BG, Speizer FE: Longitudinal and cross-sectional estimates of pulmonary function decline in neversmoking adults. Am J Epidemiol 1990, 132(4):685-700.

27. Lacson E, Ofsthun N, Lazarus JM: Effect of variability in anemia management on hemoglobin outcomes in ESRD. Am J Kidney Dis 2003, 41(1):111-124
28. Ibrahim HN, Foley RN, Zhang R, Gilbertson DT, Collins AJ: Parenteral iron use: possible contribution to exceeding target hemoglobin in hemodialysis patients. Clin J Am Soc Nephrol 2009, 4(3):623-629.

29. US Renal Data System: USRDS 2009 Annual Data Report: Atlas of EndStage Renal Disease in the United States. Bethesda, MD: National Institutes of Health, National Institute of Diabetes and Digestive and Kidney Diseases; 2009

30. Mendelssohn DC, Pisoni RL, Arrington CJ, Yeates KE, Leblanc M, Deziel C, Akiba T, Krishnan M, Fukuhara S, Lameire N, Port FK, Wolfe RA: A practicerelated risk score (PRS): a DOPPS-derived aggregate quality index for haemodialysis facilities. Nephrol Dial Transplant 2008, 23(10):3227-3233.

31. Wolfe RA, Hulbert-Shearon TE, Ashby VB, Mahadevan S, Port FK: Improvements in dialysis patient mortality are associated with improvements in urea reduction ratio and hematocrit, 1999 to 2002. Am J Kidney Dis 2005, 45(1):127-135.

32. Johnston SC: Combining ecological and individual variables to reduce confounding by indication: case study-subarachnoid hemorrhage treatment. J Clin Epidemiol 2000, 53(12):1236-1241.

\section{Pre-publication history}

The pre-publication history for this paper can be accessed here: http://www.biomedcentral.com/1471-2369/12/22/prepub

doi:10.1186/1471-2369-12-22

Cite this article as: Khan et al: Association of dialysis facility-level hemoglobin measurement and erythropoiesis-stimulating agent dose adjustment frequencies with dialysis facility-level hemoglobin variation: a retrospective analysis. BMC Nephrology 2011 12:22.

\section{Submit your next manuscript to BioMed Central and take full advantage of:}

- Convenient online submission

- Thorough peer review

- No space constraints or color figure charges

- Immediate publication on acceptance

- Inclusion in PubMed, CAS, Scopus and Google Scholar

- Research which is freely available for redistribution

Submit your manuscript at www.biomedcentral.com/submit
C Biomed Central 